\title{
Aporte del diseño gráfico en los materiales curriculares para e-Learning
}

\section{Contribution of graphic design in the curriculum materials for e-Learning}

\author{
Rebeca Lozano Castro
}

Secretaría Académica. Coordinadora de Carrera de Diseño Gráfico (Universidad Autónoma de Tamaulipas, México)

\section{Víctor García Izaguirre}

Secretario Técnico (Universidad Autónoma de Tamaulipas, México)

Fecha de recepción: 11 de enero de 2014

Fecha de revisión: 16 de enero de 2014

Para citar este artículo: Lozano Castro, R. y García Izaguirre, V. (2014):

Aporte del diseño gráfico en los materiales curriculares para e-Learning, Icono 14, volumen (12), pp. 139-155. doi: 10.7195/ri14.v12i1.648 


\section{Resumen}

Con la incursión de Tecnologías de la Información y Comunicación (TIC's) al proceso educativo, las Instituciones a nivel nacional han tenido que llevar a cabo acciones para incorporarlas al proceso de Enseñanza-Aprendizaje (E-A). Esto ha implicado generar estrategias permitiendo facilitar a los docentes la incorporación de las TIC's a la planeación de los contenidos temáticos de las asignaturas que imparten. Sin embargo, parte de ellas sólo cubren el aspecto funcional de la tecnología pero no su incorporación en el proceso y adecuación de materiales didácticos. Es necesario reconocer que pese a los esfuerzos realizados en el área educativa, las TIC's todavía no han sido potenciadas y aprovechadas. Por lo cual, es fundamental realizar un análisis para determinar la variabilidad existente entre las diversas plataformas, homologando sus diferencias mediante las premisas fundamentales del Diseño Gráfico y permitiendo la creación de materiales curriculares adaptados a estas tecnologías.

Palabras clave: Educación - Tecnología - Diseño.

\section{Abstract}

With the incursion of Information and Communication Technologies (ICT's) to the educational process, institutions at the national level have had to carry out actions to be incorporated in the teaching-learning process (E-A). This has involved generating strategies allowing teachers to facilitate the incorporation of ICT's in the planning of the thematic content of the subjects they teach. However, part of them only cover the functional aspect of the technology but not its incorporation into the process and adequacy of training materials. It is necessary to recognize that despite the efforts made in the area of education, ICT's have not yet been promoted and exploited. Therefore, it is essential to conduct an analysis to determine the variability that exists between the various platforms, aligning their differences through the fundamental premises of the Graphic Design and allowing for the creation of curricular materials adapted to these technologies.

Key Words: Education - Technology - Design.

ICONO14 | Año 2014 Volumen 12 № 1 | ISSN: 1697-8293 | DOI: ri14.v12i1.648 


\section{Introducción}

Una de las dificultades que actualmente enfrentan la mayoría de las Instituciones de educación superior (IES) en los países de habla hispana, es la poca información y capacitación que se le ha dado al profesorado en cuanto a la incorporación y uso de los materiales curriculares en los procesos educativos que involucran el uso de las Tecnologías de la Información y la Comunicación (TIC's).

Al respecto, gran parte de las Instituciones educativas se enorgullecen de contar en sus salones de clases como tecnologías de vanguardia, pero al hacer un análisis más puntual y especifico sobre su utilización, se ha demostrado que estas herramientas son subutilizadas por los profesores o definitivamente ni siquiera tienen las más mínima idea de cómo integrarla en sus procesos de enseñanza.

Todo lo anterior lleva a una inadecuada simbiosis entre la planeación que debe realizar una Institución para incorporar TIC's en sus aulas, y la capacitación que debe recibir la planta docente, que aun cuando en la mayoría de los casos se da, solo se concreta al manejo de la herramienta pero no a su integración en el currículo.

En ese sentido, el Plan Estatal de Desarrollo 2011-2016, del Estado de Tamaulipas en México, tiene un apartado que hace alusión a la Educación Integral.

En este documento, específicamente en el eje 5, su objetivo plantea "Transformar el sistema educativo para lograr la formación de ciudadanos con competencias y conocimientos para la vida y el desarrollo de la entidad, mediante el establecimiento de una nueva política educativa centrada en el aprendizaje, el fortalecimiento de la práctica docente, una coordinación eficiente y la cultura de la evaluación" y cuyas estrategias y líneas de acción establecen:

5.1.5. Fomentar la utilización eficaz de las tecnologías de información y comunicación en los procesos educativos hacia una sociedad del conocimiento.

5.1.7. Aplicar acciones complementarias en los procesos de desarrollo curricular

DOI: ri14.v12i1.648 | ISSN: 1697-8293 | Año 2014 Volumen 12 Nº 1 | ICONO14 
y contenidos educativos, que amplien las oportunidades de ingreso, permanencia y conclusión entre niveles.

5.2.1. Establecer programas de formación continua, pertinentes y de calidad que consoliden las competencias profesionales y desarrollen habilidades en el uso de tecnologías de la información y comunicación.

Todo lo anterior fundamenta el hecho de que se requiere la formación e información necesaria relacionada a la creación de los materiales curriculares adaptados a estas tecnologías, los cuales apoyarían a potenciar y fortalecer la educación superior en Tamaulipas.

Con esta intención, se vuelve necesario que la mayoría de los profesores que imparten enseñanza a nivel superior, conozcan de su existencia y el cómo utilizarlas. Para que esta situación se alcance, es imprescindible crear las condiciones favorables por medio de medidas para apoyar a los profesores, tanto para la creación de materiales curriculares como para incorporar las TIC's en sus modelos educativos.

Ahora bien, ¿cómo se relaciona todo lo anterior con la disciplina del Diseño Gráfico? A decir de quienes presentan este artículo, esta área del conocimiento puede facilitar la interpretación y utilización de las TIC's en el proceso educativo, al poder diseñar un curso taller que pueda transmitir la información necesaria para la utilización eficaz y eficiente de los materiales curriculares en el modelo educativo ELearning; donde se analice y determine la variabilidad que existen entre las diversas plataformas que son usadas para este modelo educativo en las diversas Instituciones Superior del Estado de Tamaulipas y de las cuales se conforma por la Universidad Autónoma de Tamaulipas (UAT), seis escuelas normales, cinco unidades de la Universidad Pedagógica Nacional, cinco universidades tecnológicas, siete institutos tecnológicos, tres universidades politécnicas, el Colegio San Juan Siglo XXI, el Centro de Investigación y Estudios Avanzados del Instituto Politécnico Nacional, el Centro de Biotecnología Genómica, el Centro de Investigación en Ciencia Aplicada y Tecnología Avanzada y El Colegio de Tamaulipas, así como instituciones particulares.

La intención es conformar una red de cuerpos académicos y grupos de investi- 
gadores, para la indagación del estado del arte en el uso y aplicación de materiales curriculares para los diversos modelos educativos a nivel superior en el Estado de Tamaulipas usando las premisas fundamentales que el Diseño Gráfico aporte como tal.

Para ello, este equipo de investigadores ha generado un proyecto de investigación el cual ha conformado con diferentes estudiosos y profesionales del área, mismos que han generado los objetivos de este y su planteamiento como tal. Este proyecto ha sido presentado y registrado en la Convocatoria del Programa de Estímulos a la Investigación, Desarrollo Tecnológico e Innovación 2014, realizada por la Universidad Autónoma de Tamaulipas.

El proyecto consiste en presentar una propuesta de Red Académica de Diseño Gráfico, Educación y Tecnologías, cuyo objetivo es "Generar una red de cuerpos académicos y grupos de investigadores regionales, para la indagación del estado del arte en el uso y aplicación de materiales curriculares para los diversos modelos educativos a nivel licenciatura en el Estado de Tamaulipas, que permitan plantear un curso - taller para la utilización eficaz y eficiente de éstos materiales en el modelo educativo Elearning, usando las premisas fundamentales que el Diseño Gráfico aporte como tal".

\section{Material}

En los últimos 10 años han proliferado una enorme cantidad de proyectos educativos los cuales se han enfocado a estudiar la utilización de las Tecnologías de la Información y de la Comunicación (TIC's) y sus efectos en el proceso de enseñanza - aprendizaje de las disciplinas en las cuales se han aplicado.

A decir de Cabero (2001), la Tecnología Educativa se encuentra enmarcada por tres grandes disciplinas de la ciencia de la Educación:

- La didáctica

- La Teoría curricular

- La Teoría de la enseñanza. 
Esto, se fundamenta en el hecho de que el currículum es el espacio conceptual y de intervención de la didáctica, que la didáctica es la ciencia de la enseñanza, y que la tecnología educativa se refiere al diseño de situaciones mediadas de enseñanza - aprendizaje.

Ahora bien, en relación al currículum, este se ve desde dos perspectivas fundamentales:

\section{Como espacio conceptual y de intervención que la didáctica ha de co- nocer y considerar.}

\section{Para caracterizar el contenido de la formación.}

Si bien es cierto que se han logrado enormes innovaciones en materia de Tecnología Educativa, también es cierto que ha habido ocasiones en que los resultados no son precisamente los esperados, debido en principio a que muchos tecnólogos de la educación han, escuchado y seguido el canto de las sirenas tecnológicas, tomando las soluciones de materiales curriculares que la industria ofrece y que orientan el quehacer educativo, sin haber sido estas diseñadas específicamente para un contexto en particular.

Esta proliferación de experiencias utilizando las TIC's ha demostrado que estas tecnologías no son la solución cuando no se tienen los recursos humanos y materiales necesarios que permitan su adecuada utilización. En muchos casos incluso se llega a extremos de ver las tecnologías instaladas en las escuelas sin que nadie sepa para que sirven o como se usan. Una premisa, que han demostrado fehacientemente las investigaciones efectuadas hasta el momento, es que para que una institución este realmente segura de haber integrado las tecnologías en un determinado escenario educativo, será cuando una mayoría de los profesores que imparten enseñanza en ese escenario conozcan, a título personal, cómo utilizarlas; y para que esa situación se alcance, es imprescindible crear las condiciones favorables por medio de medidas de apoyo a los profesores. Estas, pueden implicar tanto asistencia para la creación de materiales curriculares como para incorporar las TIC's en sus modelos educativos. Llevando a establecer nuevos esquemas de investigación que puedan diferenciarse en dos áreas de actuación:

ICONO14 | Año 2014 Volumen $12 N^{\circ} 1$ | ISSN: 1697-8293 | DOI: ri14.v12i1.648 


\section{La incidencia de las TIC's en la metodología de la enseñanza.}

Se trata de un campo de estudio e investigación que como cualquier otro atrae a grupos de profesores que estudian y analizan con mirada de expertos la integración de las tecnologías en la educación y van a plantear teorías, realizar investigaciones y proyectar los resultados en su entorno.

\section{El empleo de las TIC's en la enseñanza de cualquier materia.}

Entendiendo como tal, a la actividad de aplicación de las TIC's por profesores de cualquier tipo de contenido que no desean convertirse en expertos de las tecnologías y que no tienen mayor interés en el análisis del fenómeno sino en servirse de las TIC's para su labor de enseñanza sin mayores complicaciones. En este caso se encuentran la enorme mayoría de profesores de todo tipo y nivel.

La importancia de estudiar estos enfoques en que en la actualidad, gracias a las posibilidades de capacidad y flexibilidad que nos ofrecen las TIC's, se puede contemplar otro tipo de enseñanza en donde se uniría la no coincidencia espacial, con la coincidencia temporal; nueva combinación que llevará a proponer nuevos modelos de enseñanza, que propicien el acercamiento de los estudiantes a otros puntos y lugares de la instrucción, la flexibilidad en el tiempo dedicado a la instrucción, y el acercamiento a otras culturas, contextos y experiencias.

Adicional a lo anterior, también es de destacar el hecho de que actualmente el sistema educativo tiene una enorme diversidad de medios e instrumentos codificadores y presentadores de la realidad. Desde los tradicionales libros de texto y pizarra hasta la diversidad de tecnologías flexibles y sofisticadas, que van desde medios analógicos hasta digitales; desde aquellos donde el sujeto es mero receptor pasivo de sus mensajes hasta los que facilitan una interacción con el usuario, desde medios individuales hasta los multimedia, desde los que facilitan una comunicación sincrónica hasta los que lo hacen de forma asincrónica, desde aquellos donde el estudiante es mero receptor y consumidor de mensajes e informaciones a medios donde con su interacción construye su entorno significativo para el aprendizaje, y desde los que facilitan una comunicación unidireccional a los que propician verdaderos entornos colaborativos para el aprendizaje.

DOI: ri14.v12i1.648 | ISSN: 1697-8293 | Año 2014 Volumen 12 Nº 1 | ICONO14 
Toda esta diversidad de medios y potencialidades que ofrecen, no solo van a introducir formas más versátiles para su utilización y concreción, sino que repercutirán en la creación de nuevos entornos y facilidades para el aprendizaje. Estos últimos aspectos, que van desde la modificación de la interacción comunicativa unidireccional entre profesor - alumno hasta la reforma física - espacial - temporal de los escenarios de aprendizaje. Ello, sin olvidar las posibilidades que pueden proponer para el autoaprendizaje y el aprendizaje cooperativo y colaborativo entre estudiantes de diferentes contextos.

Con la incursión de las TIC's al proceso educativo, las Instituciones a nivel nacional han tenido que llevar a cabo acciones correspondientes para incorporar estas tecnologías al proceso de enseñanza - aprendizaje (E-A), las cuales han implicado generar estrategias que permitan facilitar a los docentes la incorporación de las mismas a la planeación de los contenidos temáticos de las asignaturas que imparten.

Sin embargo, gran parte de las estrategias planteadas, sólo cubren el aspecto funcional de la tecnología, pero no su incorporación en la proceso de E-A o incluso la adecuación de los materiales didácticos para ser usados mediante las TIC's.

Adicionalmente a lo anterior, la educación en México, principalmente a nivel superior, necesita plantear otros paradigmas que permitan acceder a ese tipo de formación a jóvenes que por causas sociales, políticas, geográficas, económicas 0 de otras índoles, no tienen acceso a la misma, cuestión que podría ser resuelta por la accesibilidad que ofrecen las TIC's.

Además es necesario reconocer que pese a los esfuerzos realizados, concretamente en el área educativa, las TIC's todavía no han sido potenciadas y aprovechadas en la enorme posibilidad que ofrecen para los procesos educativos.

La actividad educativa con estas tecnologías, debiera manifestarse principalmente en la creación de materiales curriculares que se adapten a las mismas, hecho que se ve reflejado en la Declaración Mundial sobre la Educación Superior en el Siglo XXI, la cual establece que "Los nuevos métodos pedagógicos también supondrán nuevos materiales didácticos". 
En ese sentido, en las escuelas a nivel Superior de la Zona Metropolitana del Sur de Tamaulipas las TIC's se han ido incorporando ya en sus procesos educativos, pero no se tiene un conocimiento puntual de la efectividad que dicha tecnología este provocando en el proceso educativo.

Las Instituciones de Educación Superior que se ubican al sur del Estado, han iniciado investigaciones sobre el uso de las TIC's en el proceso de enseñanza aprendizaje, pero estos esfuerzos aun cuando han dado resultados incipientes, son casos aislados, que requieren ser analizados desde una panorámica más holística y concordante con lo establecido en el Plan Estatal de Desarrollo 2011-2016 en su apartado de Educación Integral y que a su vez puedan ser enriquecidos por las buenas prácticas que se estén llevando a efecto en otras instituciones del país.

Caso concreto lo representa, la Facultad de Arquitectura, Diseño y Urbanismo (FADU) de la UAT quien derivado de la necesidad de optimizar su infraestructura, implemento en la primavera del 2012 un programa que permitiera llegar para el otoño del 2014 a impartir en línea entre el 15\% al 20\% de las asignaturas de sus programas de licenciatura a través de la plataforma Cisco WEBEX. Esto implico la implementación de un curso de capacitación para su profesorado en el uso del sistema, pero integrando también el aprendizaje para adecuar sus materiales didácticos a dicha tecnología. Este programa ha estado siendo monitoreado y evaluado, de manera de corregir las fallas encontradas tanto en su proceso formativo, administrativo y educativo.

Lo anterior sirvió como antecedente para establecer la necesidad de la creación de una Red de Diseño Gráfico, Educación y Tecnologías que permita establecer una colaboración estrecha ente diversas Instituciones de Educación Superior (IES), para analizar y proponer diversas acciones y estrategias que faciliten la incorporación de las TIC's en el proceso educativo.

Al ser un problema que tiene diversas vertientes y fundamentado en la experiencia de la FADU, en un principio se tomaría el determinar la creación de un Taller para el Diseño de Materiales Curriculares para E-Learning. Esto implicaría el realizar un análisis para determinar la variabilidad que existen entre las diversas 
plataformas que son usadas para este modelo educativo, tales como WEBEX, Skype, Moodle, Blackboard, entre otras; homologando sus diferencias mediante las premisas fundamentales del Diseño Gráfico.

Es decir, esta disciplina serviría como aglutinador entre las diversas TIC`s, dado que los aspectos básicos que rigen para emitir un mensaje en una plataforma tecnológica, se desempeñan en forma análoga para las otras tecnologías que tienen estructuras similares de funcionamiento.

Adicionalmente, el Diseño Gráfico impacta de manera eficaz y efectiva en la generación de materiales curriculares que debieran elaborarse para ser usados en el nuevo paradigma educativo al cual se enfrenta el proceso de enseñanza-aprendizaje.

Entre los motivos que se pueden mencionar para estudiar y analizar los medios y materiales de enseñanza, se encuentran:

1. La cantidad de medios de que se dispone ha aumentado, y sigue aumentando, considerablemente desde hace relativamente poco tiempo.

2. En contrapartida, el conocimiento que se tiene respecto a su utilización didáctica, es bastante limitado.

3. Existe poca o nula información, sobre el efecto que tienen sobre el alumnado y las expectativas que se le han asignado, como elementos potenciadores de la atención y ejecución en los estudiantes.

4. La significación que tienen para desarrollar o suplantar determinadas habilidades cognitivas de los receptores.

Todo lo anterior, apoyaría el hecho de que los medios no son exclusivamente elementos técnicos y creativos, sino más bien elementos curriculares y como tales no deben funcionar en el vacío, sino más bien en un contexto incierto y complejo como el educativo.

ICONO14 | Año 2014 Volumen 12 № 1 | ISSN: 1697-8293 | DOI: ril4.v12i1.648 
Es importante mencionar que los medios no son construcciones tecnológicas neutrales, sino asumir que al ser construcciones humanas y sociales, reflejan posiciones ideológicas tanto de forma precisa como oculta, de las personas que los diseñan y producen, y no solo con los contenidos transmitidos y sus formas de transmitirlos, sino también con la selección que se efectúa de los sistemas simbólicos que se movilizan en su producción y los contenidos que conscientemente se omiten; esto se puede ver muy puntualmente reflejado, incluso en un medio ampliamente conocido por todos, los libros de texto, quienes en gran medida reflejan la visión oficial del sistema político en turno.

Hoy en día el paisaje digital brinda ventajas a favor de las tareas docentes en materia de educación. Estas herramientas son un vínculo de información fácil y práctica para transmitir el conocimiento. Lograr servirse de los medios digitales en la vida académica permite la integración, actualización y facilita el proceso educativo en una nueva sociedad de la información.

\section{Método}

Este proyecto pretende reactivar la línea de Investigación que sobre el Diseño Gráfico Sustentable y su aplicación a la Educación, maneja el Cuerpo Académico de Diseño y Edificación Sustentable.

El método previsto para su resolución comprende una serie de etapas que se describen a continuación:

1. Realizar un censo de los programas educativos, a nivel licenciatura, que sean impartidos en la zona sur del Estado de Tamaulipas, mediante la utilización de las TIC's.

2. Evaluar la capacitación que los profesores de esos programas, han tenido en el uso de las TIC's, así como en el Diseño de materiales curriculares.

3. Determinar el muestreo representativo de los programas educativos, para poder analizar puntualmente los materiales curriculares.

4. Obtener la muestra representativa, para el análisis de los materiales curriculares que sean usados para impartir asignaturas mediante las TIC's. 
5. Evaluar los resultados obtenidos para determinar las buenas prácticas en el uso de los materiales curriculares en las TIC's.

6. Generar de la base de datos con las evaluaciones de los materiales didácticas.

7. Elaborar de la metodología para el diseño del Curso - Taller para capacitación del profesorado, a nivel licenciatura, para el uso de las TIC's.

En relación a la metodología para el diseño del Curso - Taller se considerará:

1. Determinar las áreas de oportunidad, que sean detectadas en la capacitación del profesorado en el uso de las TIC's.

2. Diseñar el plan de estudio para el Taller de capacitación para el profesorado en el uso de las TIC's.

3. Diseñar el manual de apoyo para la creación de materiales curriculares que sean aplicados al modelo educativo E-Learning.

4. Elaborar el Manual operativo y del Taller de capacitación para la creación de materiales curriculares que serán aplicados a un modelo E-learning.

\section{Resultados}

Los resultados en esta investigación aún en su fase inicial, se pueden categorizar según el impacto esperado:

- Impacto Cualitativo: La creación del Curso - Taller on-line para profesores a nivel licenciatura, permitirá establecer una metodología que permita medir la calidad para el proceso de enseñanza - aprendizaje bajo los modelos de enseñanza usando las TIC's, así como generar un modelo que sirva de punto de partida para establecer una capacitación homogénea en el uso de éstas en los programas educativos a nivel licenciatura que se imparten en el Estado de Tamaulipas, desde una perspectiva multi e interdisciplinar vinculando y haciendo concurrir complementariamente al diseño, uso de tecnologías, uso de materiales didácticos sustentables y educación, con un enfoque educativo abarcando aspectos sociales y económicos. 
- Impacto Cuantitativo: Considerando la enorme diversidad de programas educativos a nivel licenciatura que se imparten en el Estado de Tamaulipas, este modelo de capacitación puede ofrecer enormes potencialidades de crecimiento en la captación de alumnos, al incrementar los modelos educativos en que puedan impartirse estos programas, no solo de manera presencial sino a distancia y virtual.

- Impacto Científico: Generar una metodología multi e interdisciplinar que permita la capacitación del profesorado en el uso de las TIC's así como la elaboración de materiales curriculares que se deban diseñar para ser usados en esta plataforma. Esto se logrará a través del desarrollo de instrumentos de investigación generados a partir de la experiencia y conocimientos particulares de los cuerpos académicos e investigadores participantes, cuyo fin es promover una plataforma de análisis aplicable al uso de materiales curriculares para el e-learning desde una perspectiva multi e interdisciplinar vinculando y haciendo concurrir complementariamente al diseño, uso de tecnología y la educación.

- Impacto Tecnológico: El diseño y creación de una página WEB que permita ubicar el curso autodidacta así como el manual para la creación de materiales curriculares y que esté disponible a todo aquel usuario interesado en usarlo. Esta página podrá servir para fortalecer la difusión del instrumento que se genere como herramienta para medir el impacto que el proyecto tendrá en sí sobre las potencialidades de las TIC's en la educación.

- Impacto Social: Al tener el curso - taller con su manual en una página WEB con acceso libre, esta quedará a disposición de todos aquellos interesados en el uso y manejo de las TIC's para el proceso educativo. La difusión de este curso - taller incrementará en gran medida el índice de alumnos que tendrán acceso a una educación que se adecúe a sus tiempos y necesidades, logrando así incrementar la posibilidad de acceso a educación superior de estudiantes en el Estado de Tamaulipas. 
- Impacto Económico: La posibilidad de que los alumnos puedan acceder a la educación de su lugar de origen, permite que los costos de vivienda, transporte, alimentación, sean reducidos al mínimo, permitiendo así una situación económica familiar mayormente favorable. Este sistema permitirá la flexibilidad en el manejo del horario, facilitando que el alumno pueda trabajar y estudiar al mismo tiempo. En el Plan Estatal de Desarrollo del Estado de Tamaulipas se menciona que se busca ampliar las alternativas comunitarias y el acceso de los alumnos con las siguientes acciones: Fomentar la utilización eficaz de las tecnologías de información y comunicación en los procesos educativos hacia una sociedad del conocimiento; Establecer programas de formación continua, pertinentes y de calidad que consoliden las competencias profesionales y desarrollen habilidades en el uso de tecnologías de la información y comunicación.

- Impacto Ambiental: Basados en el apoyo de expertos, se buscará tener un instrumento que permita medir el impacto ambiental de un curso - taller que será difundido de manera autodidacta, sin optar por el esquema tradicional que implicaría costos de traslado, hospedaje, entre otros para la capacitación del profesorado.

Es importante destacar la importancia que el área de diseño desarrollará ante la creciente necesidad de los recursos tecnológicos como medio-método de enseñanza-aprendizaje en las nuevas generaciones de alumnos, buscando la optimización de los recursos que en ella participan.

Además de señalar la creciente y mutable modificación de los medios de enseñanza-aprendizaje a través de los medios electrónicos, señalados a través de las conferencias a distancia, programación de televisión educativa, y más recientemente el uso de la infografía como medio descriptivo de la acción a través de imágenes que muestran de manera clara y precisa la narración visual de los hechos.

La realización de este proyecto podrá unificar esfuerzos realizados y por realizar en el ámbito de la aplicación de las TIC’s en el proceso de enseñanza-aprendizaje. 


\section{Discusión}

Aun cuando la creación de la Red de Investigadores, está en su fase inicial y de autorización de los recursos requeridos en la convocatoria a la cual se inscribió, se considera que es prioritario de que se indague puntualmente sobre el estado del arte, en lo referente a la educación en el Diseño y se generen las herramientas que permitan elaborar una producción académica de mayor calidad, así como también apoyar al fortalecimiento de los Programas Educativos, resulta importante y necesaria de realizar, sobre todo si se aprovecha la coyuntura que éste este considerado como un área prioritaria en el mismo Plan Estatal de Desarrollo de Tamaulipas, lo que a su vez podría permitir pasar de una fase discursiva a establecer los elementos básicos para llevarlo a la praxis.

El conocimiento respecto al uso de las TIC's en relación al proceso de Enseñanza-Aprendizaje y de los materiales curriculares para E-Learning, se pude concluir que pese a que se cuentan con ellas en muchas de las instituciones de educación superior en el Estado de Tamaulipas, se ha detectado que no son utilizadas convenientemente por el profesorado y cuya falla principal, al menos en un análisis inicial efectuado, es debido en gran medida a la deficiente capacitación que se les da en relación a su incorporación dentro del currículo.

El que no haya materiales curriculares adecuadamente diseñados para las materias que se imparten a nivel superior, genera una problemática, que aún sin ser conclusiva en su determinación, es uno de los causales de tener una eficiencia en la calidad de la enseñanza, misma que se refleja en la falta de motivación que el alumno siente hacia el proceso en sí.

En base a ello, radica la importancia de este proyecto de Curso-Taller que apoye y permita diseñar y generar materiales curriculares, bajo los lineamientos didácticos que las TIC's requieran; cuestión que los docentes no dominan por no haber sido capacitados para adecuarse a estos nuevos formato de enseñanza.

Al tener el curso-taller con su manual en una página WEB con acceso libre, quedará a disposición de todos aquellos interesados en el uso y manejo de las TIC`s

DOI: ri14.v12i1.648 | ISSN: 1697-8293 | Año 2014 Volumen 12 Nº 1 | ICONO14 
para el proceso educativo. La difusión del mismo incrementará en gran medida el índice de alumnos que tendrán acceso a una educación que se adecúe a sus tiempos y necesidades, logrando así incrementar la posibilidad de acceso a educación superior de estudiantes en el Estado de Tamaulipas.

\section{Referencias}

Alberich, P. (2007). Comunicación Audiovisual Digital: Nuevos Medios, Nuevos, Nuevas Formas. Universidad Oberta de Catalunya. España.

ANUIES. (2004). Elementos Normativos a Considerar para los Programas de Educación Superior Impartidos en Modalidades Alternativas e la Escolarizada. 25 (4). Recuperado de http://www.anuies.mx/e_proyectos/index. php?clave=html/estrategicos1.html

Cabero, A. J., Márquez, F. D. (1999). La producción de Materiales Multimedia en la Enseñanza Universitaria. México: Ed. Kronos.

Cabero, J. (2001). Tecnología Educativa: Diseño y utilización de medios en la enseñanza. México: Ed Paidos.

Cantú Delgado, H. (2001). Desarrollo de una Cultura de Calidad. México: Ed. McGraw Hill.

Castaño, G. (2006). Las Actitudes De Los Profesores Hacia Los Medios De Enseñanza. Universidad del País Vasco. 10 (4). Recuperado de http://www.sav.us.es/pixelbit/articulos/n1/n1art/art15.htm

Chrobak, R. La metacognición y las herramientas didácticas. Universidad Nacional del Comahue. Facultad de Ingeniería, Departamento de Física. Buenos Aires 1400.8300, Neuquén. 20 (6). Recuperado de http://www.unrc. edu.ar/publicar/cde/05/Chrobak.htm

De Pablos Coello, J. M. (2007, 25 de abril). Siempre ha habido infografía. en Revista Latina de Comunicación Social 5. La Laguna Tenerife. 27 (8). Recuperado de http://www.ull.es/publicaciones/latina/a/88depablos.htm Gobierno del Estado de Tamaulipas. (2011, 10 de octubre). Plan Estatal de Desarrollo Tamaulipas 2011-2016. 10 (10). Recuperado de http://tamaulipas. gob.mx/wp-content/uploads/2011/04/Plan-Estatal.pdf

Gruffat, C. (2005). El diseño de información y la visualización del conocimiento. Argentina: Ed. Argentina. 
Ley de Ciencia y Tecnología. Cámara de Diputados. (2013). Cámara de Diputados. 10 (2). Recuperado de www.diputados.gob.mx/LeyesBiblio/doc/242.doc Mijksenaar, P. (2001). Una introducción al diseño de la información. México: Ed. Gustavo Gili.

Pérez, G. A. (2006). Diseño de Información. 24 (4). Recuperado de http://www.seth.com.mx/2006/diseno-de-informacion/

Silva, M. (2005). Educación Interactiva, Enseñanza y aprendizaje presencial y online. México: Editorial Gedisa, S. A.

Villaseñor, G. (1998). La tecnología en el proceso de enseñanza-aprendizaje. México: Editorial Trillas. 